\title{
Construction of Regular and Irregular QC-LDPC Codes: a Finite Field Approach and Masking
}

\author{
Xiaopeng Chen ${ }^{1, \mathrm{a}}$, Lin Zhou ${ }^{1,2, \mathrm{~b}}$, Rui Zhao ${ }^{1, \mathrm{c}}$ and Yucheng $\mathrm{He}^{1,2, \mathrm{~d}}$ \\ ${ }^{1}$ Xiamen Key Laboratory of Mobile Multimedia Communications, National Huaqiao University, \\ Xiamen, China \\ ${ }^{2}$ State Key Laboratory of Integrated Services Networks, Xidian University, Xi'an, China \\ a357929789@qq.com, blinzhou@hqu.edu.cn, ${ }^{\text {r } r z h a o @ h q u . e d u . c n, ~ d y u c h e n g . h e @ h q u . e d u . c n ~}$
}

Keywords: LDPC; masking; Quasi-Cyclic

Abstract. Quasi-Cyclic Low-Density Parity-Check (QC-LDPC) codes require less hardware resources due to the quasi-cyclic structure of parity-check matrices. In this paper, two improved methods for constructing QC-LDPC codes are proposed. Firstly, in order to make sure the masked LDPC codes have flexible code rate and code length, an improved construction of masking matrix is presented. The proposed regular masking matrix of QC-LDPC codes have the girth at least 6 . Then, the irregular codes are constructed by using improved finite field approach and PEG algorithm, which result in large girth and less short cycles. Simulations demonstrate that the proposed QC-LDPC codes have both lower error floor and good waterfall performance.

\section{Introduction}

Low-density parity-check (LDPC) codes were discovered by Gallager in 1962[1], and then rediscovered in the late 1990s. They were proved to be channel-capacity-approaching codes[2]. Since then, various methods for constructing LDPC codes have been proposed.

The construction of LDPC codes can be divided into two categories: random codes[3] and structured codes[4,5]. Random codes have good performance, but it's too complex to application. Structured codes with optimized design can not only performance as well as random codes, but also get lower complexity in practical applications.

Recently, algebraic method have became an effective method to construct structured codes. This method can obtain the codes with good error performance more easily. Based on the algebraic construction of LDPC codes that have quasi-cyclic structure, called QC-LDPC codes[6,7]. In order to improve the bit error rate(BER) performance of QC-LDPC codes, many methods have been proposed to optimize the girth, degree distribution, the trapping set and so on. To improve the error floor performance, masking technique[8] was proposed to construction QC-LDPC codes.

In this paper, we present constructions of both regular and irregular QC-LDPC codes with large girth. First, a general construction based on finite field and masking technology is introduced. Second, an improved construction method of masking matrix of QC-LDPC codes is proposed. This method can obtain codes with a wider range of rate and also have a good performance. Then, we propose an improved method for designing base matrix by using two subsets in the finite field. Based on this base matrix, we can obtain irregular codes via masking by PEG algorithm[9]. At last, the simulation results prove that our methods have good error performance.

\section{QC-LDPC Codes Based on Finite Field and Masking}

Construction of base matrix. In general, QC-LDPC codes based on finite field need to construct an $m \times n$ base matrix $\boldsymbol{B}$. It is shown in Eq. 1: 


$$
B=\left[\begin{array}{cccc}
w_{1,1} & w_{1,2} & \cdots & w_{1, n} \\
w_{2,1} & w_{2,2} & \cdots & w_{2, n} \\
\vdots & \vdots & \ddots & \vdots \\
w_{m, 1} & w_{m, 2} & \cdots & w_{m, n}
\end{array}\right]
$$

where $w_{i, j}(1 \leq i \leq m, 1 \leq j \leq n)$ is an element of Galois field $\mathrm{GF}(\mathrm{q})$. Matrix $\boldsymbol{B}$ satisfied the row-column constraint: any two rows(or two columns) have at most one same non-zero elements at the same place. This constraint ensures that the constructed codes have girth at least 6 .

Let $\boldsymbol{A}_{b}\left(w_{i, j}\right)$ be a $(\mathrm{q}-1) \times(\mathrm{q}-1)$ circulant permutation matrix if $w_{i, j} \neq 0$, and it's a (q-1)×(q-1) zero matrix if $w_{i, j}=0$. By replacing each $w_{i, j}$ with $\boldsymbol{A}_{b}\left(w_{i, j}\right)$, we obtained a $m(\mathrm{q}-1) \times n(\mathrm{q}-1)$ parity matrix $\boldsymbol{H}$.

Construction via masking. Let $\boldsymbol{Z}=\left[z_{i, j}\right]$ be a $m \times n$ masking matrix, where $1 \leq i \leq m, 1 \leq j \leq n, z_{i, j} \in$ $\{0,1\}$, the masked matrix $\boldsymbol{M}$ is shown in Eq. 2:

$$
M=Z \Theta H=\left[\begin{array}{cccc}
z_{1,1} A_{b}\left(w_{1,1}\right) & z_{1,2} A_{b}\left(w_{1,2}\right) & \cdots & z_{1, n} A_{b}\left(w_{1, n}\right) \\
z_{2,1} A_{b}\left(w_{2,1}\right) & z_{2,2} A_{b}\left(w_{2,2}\right) & \cdots & z_{2,1} A_{b}\left(w_{2, n}\right) \\
\vdots & \vdots & \ddots & \vdots \\
z_{m, 1} A_{b}\left(w_{m, 1}\right) & z_{m, 2} A_{b}\left(w_{m, 2}\right) & \cdots & z_{m, n} A_{b}\left(w_{m, n}\right)
\end{array}\right]
$$

where $z_{i, j} \boldsymbol{A}_{b}\left(w_{i, j}\right)=\boldsymbol{A}_{b}\left(w_{i, j}\right)$ if $z_{i, j}=1, z_{i, j} \boldsymbol{A}_{b}\left(w_{i, j}\right)=\mathbf{0}$ if $z_{i, j}=0$. The masked matrix $\boldsymbol{M}$ gets the same degree distribution as the masking matrix $Z$. So the masking matrix plays a significant part in the performance of the masked LDPC codes.

\section{Construction of Regular and Irregular Codes via the Proposed Method}

The improved Construction of regular codes. In [10], a (3,6)-regular masking matrix is shown in Eq. 3:

$$
Z(4,8)=\left[\begin{array}{llllllll}
1 & 0 & 1 & 0 & 1 & 1 & 1 & 1 \\
0 & 1 & 0 & 1 & 1 & 1 & 1 & 1 \\
1 & 1 & 1 & 1 & 1 & 0 & 1 & 0 \\
1 & 1 & 1 & 1 & 0 & 1 & 0 & 1
\end{array}\right]
$$

The girth of the regular QC-LDPC codes increased by his masking matrix from 6 to 8, and result in a masked matrix $\boldsymbol{M}$ with row weight 6 and column weight 3, respectively. It is very effective in decreasing short cycles and increasing the girth, so it has a good BER performance and low error floor. But this masking matrix $\mathbf{Z}(4,8)$ only suit condition in fixed size and code rate of base matrix.

To solve the deficiency of this masking matrix, we proposed an improved masking matrix as follows: repeat the first pair of the columns and third pair of columns in $Z(4,8) p$ times, obtain $Z(4,8 p)$ with $4 \times 8 k$ size. Then downward expand $Z(4,8 p) t$ times, obtain $Z(4 t, 8 p)$ with $4 t \times 8 p$ size.

Let $\boldsymbol{R}=\boldsymbol{Z}(4,8)$, improved masking matrix $\mathbf{Z}(4 t, 8 p)$ is shown in Eq. 4 :

$$
Z(4 t, 8 p)=\left[\begin{array}{cccc}
R_{1,1} & R_{1,2} & \cdots & R_{1, p} \\
R_{2,1} & R_{2,2} & \cdots & R_{2, p} \\
\vdots & \vdots & \ddots & \vdots \\
R_{t, 1} & R_{t, 1} & \cdots & R_{t, p}
\end{array}\right]
$$


where $t \geq 1, p \geq 1$. Notice that the masking matrix $Z(4 t, 8 p)$ also satisfied the $3 \times 3 \mathrm{SM}$-constraint in [10], so the masked matrices have the girth- 8 at least, which result in good BER performance and low error floor.

The improved Construction of irregular codes. To construct base matrix, let $\alpha$ be the primitive element of GF(q). For $1 \leq m, n \leq \mathrm{q}$, let $S_{1}=\left\{\alpha^{k_{0}}, \alpha^{k_{1}}, \ldots, \alpha^{k_{m-1}}\right\}, S_{2}=\left\{\alpha^{l_{0}}, \alpha^{l_{1}}, \ldots, \alpha^{l_{n-1}}\right\}$ be two subsets of GF(q), we can obtain matrix element $w_{i, j}$ in base matrix $\boldsymbol{B}$, which is shown in Eq. 5:

$$
w(i, j)=\alpha^{k_{i}}+\alpha^{I_{j}}
$$

where $0 \leq k_{i} \leq m-1,0 \leq l_{j} \leq n-1$. With different choices of two subsets $\mathrm{S}_{1}$ and $\mathrm{S}_{2}$, we can obtain different base matrix $\boldsymbol{B}$ with girth 6 or 8. In order to obtain irregular codes, we use PEG algorithm to construct masking matrix. By using degree distribution optimization, a proper masking matrix can be obtained. This method has better BER performance compared with the traditional construction by using PEG algorithm.

\section{Simulation Results}

The performance of different codes constructed by our methods are verified for additive white Gaussian noise channel. Codes are modulated by BPSK and decoded by sum-product algorithm. Maximum 50 iterations are considered for iterative decoding.

Example 1: First, we construct base matrix $\boldsymbol{B}(6,121)$ over $\mathrm{GF}(128)$. The masking matrix $\mathbf{Z}(6,121)$ was constructed according to Eq.4. By masking $\boldsymbol{B}(6,121)$ with $\boldsymbol{Z}(6,121)$, we obtained a masked matrix $\boldsymbol{M}(6,121)$. Every elements in $\boldsymbol{M}(6,121)$ can be replaced by $127 \times 127$ permutation matrices. Finally, we obtained a $(6,121)$-regular $(15367,14605)$ QC-LDPC code $\mathrm{C}_{1}$ with rate 0.95 . The BER performance of this code is shown in Fig. 1. It shows that the performance of the code $\mathrm{C}_{1}$ is much better than the code $C_{2}(16120,15345)$ given in [10]. The code $C_{1}$ outperforms the code $C_{2}$ by about $0.25 \mathrm{~dB}$ at $\mathrm{BER}=1 \times 10^{-5}$.

Example 2: In this example, let GF(128) be the field for code construction. Based on this field, we construct a $4 \times 28$ base matrix $\boldsymbol{B}(4,28)$. Masking matrix $\boldsymbol{Z}(4,28)$ is given according to Eq. 4 . By using masking technology, we can get the masked matrix $\boldsymbol{M}(4,28)$ with row weight 21 and column weight 3 . Hence the null space of $\mathbf{Z}(4,28)$ gives a $(3556,3048)$ regular QC-LDPC code $\mathrm{C}_{3}$ with rate 0.86 . For comparison, a corresponding regular $(3584,3072)$ code $C_{4}$ is given in [11]. The error performance is shown in Fig. 2. We can see that the error performance of $\mathrm{C}_{3}$ is better than that of the code $\mathrm{C}_{4}$ at all SNR region. It shows that the code construct with improved masking matrix has good BER performance and low error floor.

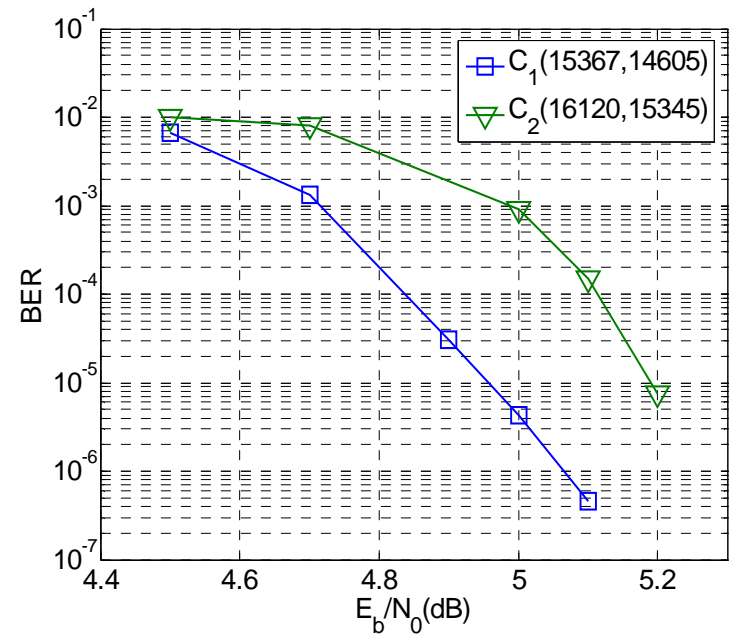

Fig. 1. The error performance of the codes $C_{1}$ given in Example 1 and $\mathrm{C}_{2}$ given in [10].

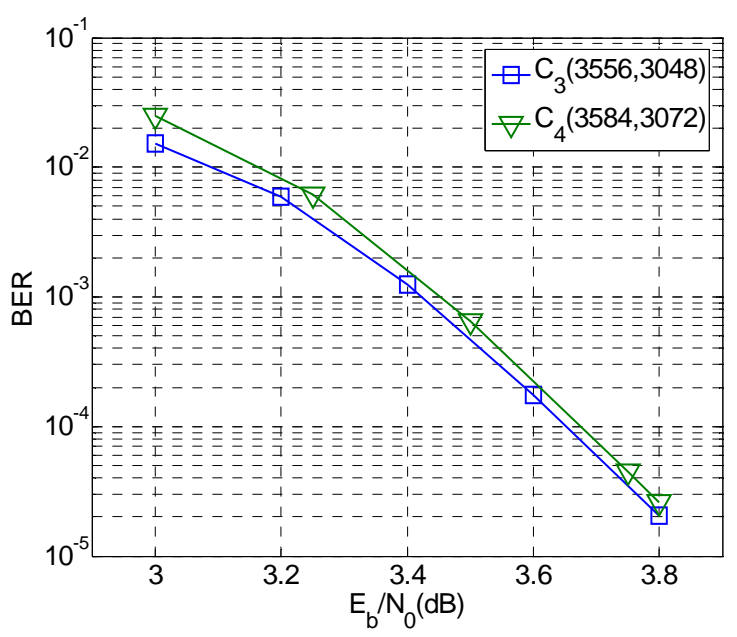

Fig. 2. The error performance of the codes $\mathrm{C}_{3}$ given in Example 2 and $\mathrm{C}_{4}$ given in [11]. 
Example 3: In this example, we construct an irregular QC-LDPC code by using two finite field subsets and PEG masking matrix. Let $\alpha$ be a primitive element of GF(64), we choose $S_{1}=\left\{\alpha^{1}, \alpha^{2}, \ldots, \alpha^{15}\right\}$ and $S_{2}=\left\{\alpha^{22}, \alpha^{23}, \ldots, \alpha^{51}\right\}$ be two subsets of elements, form a $15 \times 30$ base matrix $\boldsymbol{B}(15,30)$. Next, the $15 \times 30$ masking matrix $\boldsymbol{Z}(15,30)$ was constructed by PEG algorithm with degree distribution $\lambda(x)=\mathbf{0 . 4 6} x+\mathbf{0 . 1 2} x^{2}+\mathbf{0 . 1 2} x^{3}+\mathbf{0 . 2 3} x^{4}+\mathbf{0 . 0 7} x^{14}$, then we obtained a masked matrix $\boldsymbol{M}(15,30)$. The null space of $\boldsymbol{M}(15,30)$ gives an irregular $(1890,945)$ code $\mathrm{C}_{5}$ with rate 0.5 . For comparison, an irregular $(1890,945)$ code $\mathrm{C}_{6}$ is given in[12]. The BER performance is shown in Fig. 3. At the BER of $10^{-6}$, code $\mathrm{C}_{5}$ outperforms the code $\mathrm{C}_{6}$ by about $0.1 \mathrm{~dB}$. The code constructed by two subsets performs slightly better than finite field approach in [12].

Example 4: Again, consider the field GF(64) for code construction. Set $S_{1}=\left\{\alpha^{1}, \alpha^{2}, \ldots, \alpha^{16}\right\}$, $S_{2}=\left\{\alpha^{23}, \alpha^{24}, \ldots, \alpha^{54}\right\}$ be two subsets, form a $16 \times 32$ base matrix $\boldsymbol{B}(16,32)$. The masking matrix $Z(16,32)$ was constructed by PEG algorithm with degree distribution $\lambda(x)=\mathbf{0 . 4 6} x+\mathbf{0 . 1 2} x^{2}+\mathbf{0 . 1 2} x^{3}+\mathbf{0 . 2 3} x^{4}+\mathbf{0 . 0 7} x^{14}$. The null space of masked matrix $\boldsymbol{M}(16,32)$ gives an irregular $(2016,1008)$ code $C_{7}$ with rate 0.5 . The code performance is shown in Fig. 4 . Also included in Fig. 4 is the BER performance of a $(2016,1008)$ code $\mathrm{C}_{8}$ given in [13]. It shows that the two codes perform the same before SNR of 2.0, but the code $\mathrm{C}_{7}$ outperforms the $\mathrm{C}_{8}$ starting from the SNR of 2.0.

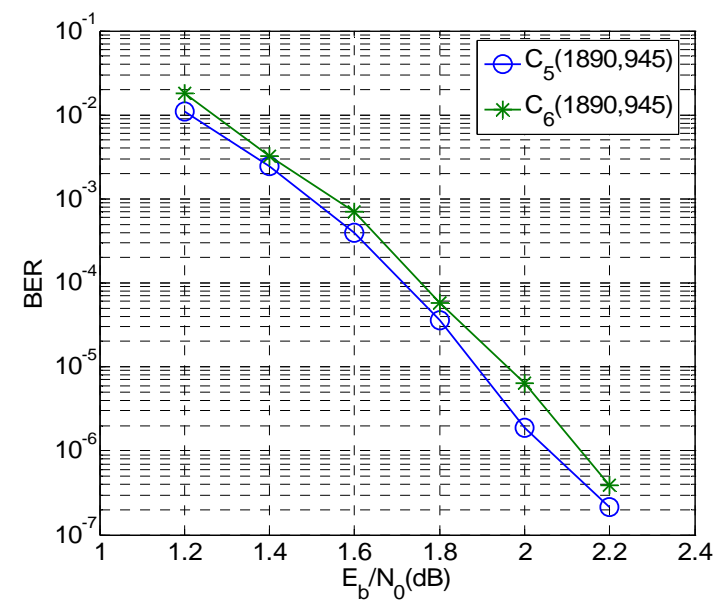

Fig.3. The error performance of the codes $\mathrm{C}_{5}$ given in Example 3 and $\mathrm{C}_{6}$ given in [12].

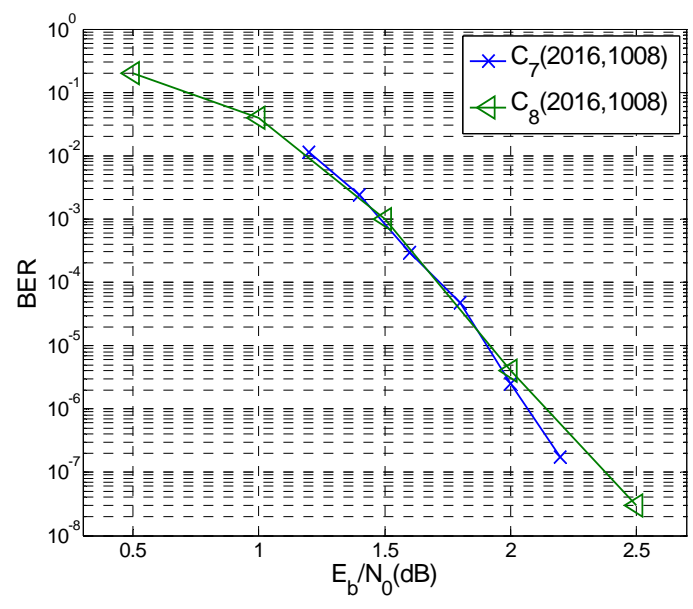

Fig.4. The error performance of the codes $\mathrm{C}_{7}$ given in Example 4 and $\mathrm{C}_{8}$ given in [13].

\section{Conclusions}

In this paper, we firstly proposed an improved method for construct masking matrix with large girth and low error floor. Based on the improved masking matrix which satisfies the $3 \times 3 \mathrm{SM}$-constraint, the regular QC-LDPC codes with girth-8 were constructed, it has flexible code rate and good error performance compared to method in [10]. Moreover, we proposed two element subsets in finite field to construct the irregular QC-LDPC codes via PEG algorithm. The construction obeys the row-column constraints, which ensures that the girth is at least 6 . Simulation results demonstrate that the proposed construction approaches of QC-LDPC codes have better BER performance and low error floor than some existing methods.

\section{Acknowledgements}

This work was supported in part by the grants from the National Natural Science Foundation of China (61302095, 61401165), the Natural Science Foundation of Fujian Province of China (2014J05076, 2014J01243, 2015J01262), the Science Foundation of National Huaqiao University (12BS219, 
13Y0384, 13BS101), and the Graduate Student Scientific Research Innovation Ability Cultivation Plan Projects of Huaqiao University under the Grant 1400201028.

\section{References}

[1] R. G. Gallager, "Low-density parity-check codes," IRE Transactions on Information Theory, IT-8, pp. 21-28, Jan. 1962.

[2] D. J. C. MacKay and R. M. Neal, "Near Shannon limit performance of low density parity-check codes," Electron. Lett., vol. 32, no. 18, pp. 1645-1646, Aug. 1996.

[3] S. Y. Chung, G. D. Forney, T. Richardson, and R. Urbanke, "On the design of low-density parity-check codes within $0.0045 \mathrm{~dB}$ of the Shannon limit," IEEE Commun. Lett., vol. 5, no. 2, pp. 58-60, Feb. 2001.

[4] Y. Kou, S. Lin, and M. Fossorier, "Low density parity check codes based on finite geometries: A rediscovery and new results," IEEE Trans. Inf. Theory, vol. 47, no. 7, pp. 2711-2736, Nov. 2001.

[5] Y. Kou, S. Lin, and M. Fossorier, "Construction of low density parity check codes: A geometric approach," in Proc. 2nd Int. Symp. Turbo Codes and Related Topics, Brest, France, Sep. 2000, pp. 137-140.

[6] L. Chen, J. Xu, I. Djurdjevic, and S. Lin, "Near-Shannon-limit quasicyclic low-density parity-check codes," IEEE Trans. Commun., vol. 52, no. 7, pp. 1038-1042, Jul. 2004.

[7] M. P. C. Fossorier, "Quasi-cyclic low-density parity-check codes from circulant permutation matrices," IEEE Trans. Inf. Theory, vol. 50, no. 8, pp. 1788-1793, Aug. 2004.

[8] J. Xu, L. Chen, I. Djurdjevic, S. Lin, and K. Abdel-Ghaffar, "Construction of regular and irregular LDPC codes: geometry decomposition and masking," IEEE Trans. Commun., vol. 53, no. 1, pp. 121-134, Jan. 2007.

[9] X.-Y. Hu, E. Eleftheriou, and D.-M. Arnold, "Progressive edge-growth Tanner graphs," in Proc. IEEE GLOBECOM 2001, San Antonio, TX, Nov. 2001, pp. 995-1001.

[10]J. Li, K. Liu, S. Lin, and K. Abdel-Ghaffar, "Algebraic quasi-cyclic LDPC codes: Construction, low error-floor, large girth and a reduced-complexity decoding scheme," IEEE Trans. Commun., vol. 62, no. 8, pp. 2626-2637, Aug. 2014.

[11]Vafi S, Majid N R. , “A New Scheme of High Performance Quasi-Cyclic LDPC Codes With Girth 6,” IEEE Commun. Lett., vol. 19, no. 10, pp. 1666-1669, Feb. 2015.

[12]L. Lan et al., "Construction of quasi-cyclic LDPC codes for AWGN and binary erasure channels: A finite field approach,” IEEE Trans. Inf. Theory, vol. 53, no. 7, pp. 2429-2458, Jul. 2007.

[13]Zongcheng Li, "Design and implementation of the rate-compatible QC-LDPC codes,"[D]. Xidian University, 2011. (In Chinese) 